\title{
EXISTENCE RESULTS FOR A PERTURBED FOURTH-ORDER EQUATION
}

\author{
MOHAMMAD REZA HEIDARI TAVANI ${ }^{1}$ \\ ${ }^{1}$ Department of Mathematics, \\ Ramhormoz branch, Islamic Azad University, Ramhormoz, Iran \\ m.reza.h56@gmail.com
}

\begin{abstract}
The existence of at least three weak solutions for a class of perturbed fourth-order problems with a perturbed nonlinear term is investigated. Our approach is based on variational methods and critical point theory.

Key words and Phrases: Fourth-order equation, weak solution, critical point theory, variational methods.
\end{abstract}

\begin{abstract}
Abstrak. Paper ini memeriksa keujudan sekurang-kurangnya tiga solusi lemah dari kelas masalah orde ke-empat dipertubasi dengan sebuah suku non linier di pertubasi. Pendekatan yang kami lakukan berdasarkan metode variasional dan teori titik kritis. Kata kunci: Persamaan orde ke-empat, solusi lemah, teori titik kritis, metode variasional.
\end{abstract}

\section{INTRODUCTION}

Consider the following fourth-order problem

$$
\left\{\begin{array}{l}
u^{(i v)}(x)=\lambda f(x, u(x))+h(u(x)), \quad x \in[0,1], \\
u(0)=u^{\prime}(0)=0 \\
u^{\prime \prime}(1)=0, \quad u^{\prime \prime \prime}(1)=\mu g(u(1)),
\end{array}\right.
$$

where $\lambda$ and $\mu$ are a positive parameters, $f:[0,1] \times \mathbb{R} \rightarrow \mathbb{R}$ is $L^{1}$-Carathéodory function, $g: \mathbb{R} \rightarrow \mathbb{R}$ is a continuous function and $h: \mathbb{R} \rightarrow \mathbb{R}$ is a Lipschitz continuous function with the Lipschitz constant $0<L<1$, i.e.,

$$
\left|h\left(t_{1}\right)-h\left(t_{2}\right)\right| \leq L\left|t_{1}-t_{2}\right|
$$

for every $t_{1}, t_{2} \in \mathbb{R}$, and $h(0)=0$. According to definition of function $h$ we see that

$$
|h(t)| \leq L|t|
$$

2000 Mathematics Subject Classification: 34B15, 58E05.

Received: 7 May 2017, revised: 21 June 2017, accepted: 21 June 2017. 
for each $t \in \mathbb{R}$.

The problem (1) is related to the deflections of elastic beams based on nonlinear elastic. In relation with the problem (1), there is an interesting physical description. An elastic beam of length $d=1$, which is clamped at its left side $x=0$, and resting on a kind of elastic bearing at its right side $x=1$ which is given by $\mu g$. Along its length, a load $\lambda f+h$, is added to cause deformations. if $u=u(x)$ denotes the configuration of the deformed beam, then since $u^{\prime \prime \prime}(1)$ represents the shear force at $x=1$, the condition $u^{\prime \prime \prime}(1)=\mu g(u(1))$ means that the vertical force is equal to $\mu g(u(1))$, which denotes a relation, possibly nonlinear, between the vertical force and the displacement $u(1)$.

Classical bending theory of elastic beams are very important in engineering sciences and so many studies have been done on a variety of problems like this.

For example in [12], authors considered iterative solutions for problem (1) in the case of $\lambda=\mu=1$ with nonlinear boundary conditions. In $[9,11]$ Some authors by using the critical point theory studied Existence and multiplicity results for this kind of problem . In particular, by using a variational methods the existence of non-zero solutions for problem (1) in the case of $h(t) \equiv 0$ has been established in $[4]$.

In the present paper, using one kind of three critical points theorem obtained in [5] which we recall in the next section, we establish the existence of at least three weak solutions for the problem (1). For example this theorem used to ensure the existence of at least three solutions for perturbed boundary value problems in the papers $[2,3,7,10]$.

\section{Preliminaries}

Our main tools is the following three critical points Theorem. In this following Theorem the coercivity of the functional $\Phi-\lambda \Psi$ is required.

Theorem 2.1 ([5], Theorem 2.6). Let $X$ be a reflexive real Banach space, $\Phi: X \longrightarrow$ $\mathbb{R}$ be a coercive continuously Gâteaux differentiable and sequentially weakly lower semicontinuous functional whose Gâteaux derivative admits a continuous inverse on $X^{*}, \Psi: X \longrightarrow \mathbb{R}$ be a continuously Gâteaux differentiable functional whose Gâteaux derivative is compact such that $\Phi(0)=\Psi(0)=0$.

Assume that there exist $r>0$ and $w \in X$, with $r<\Phi(w)$ such that

$$
\left.\left(a_{1}\right) \frac{\sup _{\Phi(u) \leq r} \Psi(u)}{r}<\frac{\Psi(w)}{\Phi(w)},\left(a_{2}\right) \text { for each } \lambda \in \Lambda_{r}:=\right] \frac{\Phi(w)}{\Psi(w)}, \frac{r}{\sup _{\Phi(u) \leq r} \Psi(u)}[
$$

the functional $\Phi-\lambda \Psi$ is coercive.

Then, for each $\lambda \in \Lambda_{r}$ the functional $\Phi-\lambda \Psi$ has at least three distinct critical points in $X$.

Let us recall some basic concepts. Denote

$$
X:=\left\{u \in H^{2}([0,1]) \mid u(0)=u^{\prime}(0)=0\right\},
$$


where $H^{2}([0,1])$ is the sobolev space of all functions $u:[0,1] \rightarrow \mathbb{R}$ such that $u$ and its distributional derivative $u^{\prime}$ are absolutely continuous and $u^{\prime \prime}$ belongs to $L^{2}([0,1])$.It is clearly that $X$ is a Hilbert space with the usual norm

$$
\|u\|_{X}=\left(\int_{0}^{1}\left(\left|u^{\prime \prime}(x)\right|^{2}+\left|u^{\prime}(x)\right|^{2}+|u(x)|^{2}\right) d x\right)^{1 / 2}
$$

which is equivalent to the norm

$$
\|u\|=\left(\int_{0}^{1}\left(\left|u^{\prime \prime}(x)\right|^{2} d x\right)^{1 / 2} .\right.
$$

Also according to [13] the embedding $X \hookrightarrow C^{1}([0,1])$ is compact and we have

$$
\|u\|_{C^{1}([0,1])}=\max \left\{\|u\|_{\infty},\left\|u^{\prime}\right\|_{\infty}\right\} \leq\|u\|
$$

for each $u \in X$. We suppose that the Lipschitz constant $L$ of the function $h$ satisfies $L<1$. A function $u:[0,1] \rightarrow \mathbb{R}$ is a weak solution to the problem (1) if $u \in X$ and $\int_{0}^{1} u^{\prime \prime}(x) v^{\prime \prime}(x) d x-\lambda \int_{0}^{1} f(x, u(x)) v(x) d x-\mu g(u(1)) v(1)-\int_{0}^{1} h(u(x)) v(x) d x=0$ for every $v \in X$. Standard methods (see [13, Lemma 2.1]) show that if $f$ is continuous, then each weak solution $u$ of the problem (1) is a classical solution.

Put

and

$$
\begin{gathered}
F(x, t)=\int_{0}^{t} f(x, \xi) d \xi \quad \text { for all }(x, t) \in[0,1] \times \mathbb{R}, \\
G(t)=\int_{0}^{t} g(\xi) d \xi \quad \text { for all } t \in \mathbb{R}, \\
G^{\beta}=\max _{|t| \leq \beta} G(t) \quad \text { for all } \beta>0,
\end{gathered}
$$

$$
H(t)=\int_{0}^{t} h(\xi) d \xi \quad \text { for all } t \in \mathbb{R} .
$$

Now we express the following proposition that, in the proof of the main theorem of this paper is required.

Proposition 2.2. Let $T: X \rightarrow X^{*}$ be the operator defined by

$$
T(u)(v)=\int_{0}^{1} u^{\prime \prime}(x) v^{\prime \prime}(x) d x-\int_{0}^{1} h(u(x)) v(x) d x
$$

for every $u, v \in X$. Then $T$ admits a continuous inverse on $X^{*}$.

Proof. For every $u, v \in X$ we have,

$\langle T(u)-T(v), u-v\rangle \geq \int_{0}^{1}\left|u^{\prime \prime}(x)-v^{\prime \prime}(x)\right|^{2} d x-L \int_{0}^{1}|u(x)-v(x)|^{2} d x \geq(1-L)\|u-v\|^{2}$.

So $T$ is uniformly monotone. By [[14], Theorem 26.A(d)], we have that $T^{-1}$ exists and is continuous. 


\section{Main Results}

In order to introduce our first result, fixing two positive constants $\theta$ and $\delta$ such that

$$
8 \pi^{4}(1+L)\left(\frac{2}{3}\right)^{3} \delta^{2} \int_{0}^{1} \sup _{|t| \leq \theta} F(x, t) d x<(1-L) \theta^{2} \int_{\frac{3}{4}}^{1} F(x, \delta) d x,
$$

and taking

$$
\lambda \in \Lambda:=] \frac{4(1+L)\left(\frac{2}{3}\right)^{3} \pi^{4} \delta^{2}}{\int_{\frac{3}{4}}^{1} F(x, \delta) d x}, \frac{(1-L) \theta^{2}}{2 \int_{0}^{1} \sup _{|t| \leq \theta} F(x, t) d x}[,
$$

and set $\eta_{\lambda, g}$ given by

$\eta_{\lambda, g}:= \begin{cases}\frac{(1-L) \theta^{2}-2 \lambda \int_{0|t| \leq \theta}^{1} \sup _{|t|} F(x, t) d x}{2 G^{\theta}} & \text { if } G(\delta) \geq 0 \\ \min \left\{\frac{(1-L) \theta^{2}-2 \lambda \int_{0}^{1} \sup _{|t| \leq \theta} F(x, t) d x}{2 G^{\theta}}, \frac{4(1+L) \pi^{4} \delta^{2}-\left(\frac{3}{2}\right)^{3} \lambda \int_{\frac{3}{4}}^{1} F(x, \delta) d x}{\left(\frac{3}{2}\right)^{3} G(\delta)}\right\} & \text { if } G(\delta)<0 .\end{cases}$

It is easy to show that $\eta_{\lambda, g}>0$ (also see [4]) . Now we express the main results.

Theorem 3.1. Assume that there exist two positive constants $\theta$ and $\delta$ with $\theta<$ $\frac{8 \pi^{2}}{3 \sqrt{3}} \delta$, such that

$\left(A_{1}\right) F(x, t) \geq 0$, for each $(x, t) \in\left[0, \frac{3}{4}[\times[0, \delta]\right.$;

$\left(A_{2}\right) 8 \pi^{4}(1+L)\left(\frac{2}{3}\right)^{3} \delta^{2} \int_{0}^{1} \sup _{|t| \leq \theta} F(x, t) d x<(1-L) \theta^{2} \int_{\frac{3}{4}}^{1} F(x, \delta) d x$;

$\left(A_{3}\right) \lim \sup _{|t| \rightarrow+\infty} \frac{\sup _{x \in[0,1]} F(x, t)}{t^{2}} \leq 0$.

Then, for each $\lambda \in \Lambda$ and for every continuous function $g: \mathbb{R} \rightarrow \mathbb{R}$ there exists $\eta_{\lambda, g}>0$ given by (5) such that, for each $\left.\mu \in\right] 0, \eta_{\lambda, g}[$, the problem (1) admits at least three weak solutions in $X$.

Proof. Fix $\lambda \in \Lambda$ and $\mu \in] 0, \eta_{\lambda, g}[$. Let $\Phi, \Psi: X \rightarrow \mathbb{R}$ be defined by

$$
\Phi(u)=\frac{1}{2} \int_{0}^{1}\left|u^{\prime \prime}(x)\right|^{2} d x-\int_{0}^{1} H(u(x)) d x
$$


and

$$
\Psi(u)=\int_{0}^{1} F(x, u(x)) d x+\frac{\mu}{\lambda} G(u(1))
$$

for every $u \in X$. It is well known that $\Psi$ is a differentiable functional whose differential at the point $u \in X$ is

$$
\Psi^{\prime}(u)(v)=\int_{0}^{1} f(x, u(x)) v(x) d x+\frac{\mu}{\lambda} g(u(1)) v(1),
$$

as well as is sequentially weakly upper semicontinuous. Furthermore, $\Psi^{\prime}: X \rightarrow X^{*}$ is a compact operator[see [13]-page 1602]. Moreover, $\Phi$ is continuously differentiable whose differential at the point $u \in X$ is

$$
\Phi^{\prime}(u)(v)=\int_{0}^{1} u^{\prime \prime}(x) v^{\prime \prime}(x) d x-\int_{0}^{1} h(u(x)) v(x) d x
$$

for every $v \in X$, while Proposition 2.2 gives that $\Phi^{\prime}$ admits a continuous inverse on $X^{*}$. Furthermore, $\Phi$ is sequentially weakly lower semicontinuous. Put

$$
r:=\frac{(1-L)}{2} \theta^{2}
$$

and

$$
w(x):= \begin{cases}0 & \text { if } x \in\left[0, \frac{3}{8}\right] \\ \delta \cos ^{2}\left(\frac{4 \pi x}{3}\right) & \text { if } x \in] \frac{3}{8}, \frac{3}{4}[ \\ \delta & \text { if } x \in\left[\frac{3}{4}, 1\right] .\end{cases}
$$

We clearly observe that $w \in X$ and,

$$
\|w\|^{2}=8 \pi^{4} \delta^{2}\left(\frac{2}{3}\right)^{3} .
$$

Now according to (2) and definition of functional $\Phi$ we have,

and in particular,

$$
\frac{(1-L)}{2}\|u\|^{2} \leq \Phi(u) \leq \frac{(1+L)}{2}\|u\|^{2}
$$

$$
4(1-L) \pi^{4} \delta^{2}\left(\frac{2}{3}\right)^{3} \leq \Phi(w) \leq 4(1+L) \pi^{4} \delta^{2}\left(\frac{2}{3}\right)^{3} .
$$

Now using $\theta<\frac{8 \pi^{2}}{3 \sqrt{3}} \delta$, and (10) we observe that

$$
0<r<\Phi(w) .
$$

Since $\frac{(1-L)}{2}\|u\|^{2} \leq \Phi(u)$, for each $u \in X$, we see that

$$
\begin{aligned}
\left.\left.\Phi^{-1}(]-\infty, r\right]\right) & =\{u \in X ; \Phi(u) \leq r\} \\
& =\left\{u \in X ; \frac{(1-L)}{2}\|u\|^{2} \leq r\right\} \\
& \subseteq\{u \in X ;|u(x)| \leq \theta \text { for each } x \in[0,1]\},
\end{aligned}
$$


and it follows that

$$
\begin{aligned}
\sup _{\left.\left.u \in \Phi^{-1}(]-\infty, r\right]\right)} \Psi(u) & =\sup _{\left.\left.u \in \Phi^{-1}(]-\infty, r\right]\right)}\left(\int_{0}^{1} F(x, u(x)) d x+\frac{\mu}{\lambda} G(u(1))\right) \\
& \leq \int_{0}^{1} \sup _{|t| \leq \theta} F(x, t) d x+\frac{\mu}{\lambda} G^{\theta} .
\end{aligned}
$$

On the other hand, in view of $\left(A_{1}\right)$, since $w(x) \in[0, \delta]$ for each $x \in[0,1]$, we have

$$
\begin{aligned}
\Psi(w) & =\int_{0}^{1} F(x, w(x)) d x+\frac{\mu}{\lambda} G(w(1)) \\
& \geq \int_{\frac{3}{4}}^{1} F(x, \delta) d x+\frac{\mu}{\lambda} G(\delta) .
\end{aligned}
$$

Hence, we have

$$
\frac{\sup _{\left.\left.u \in \Phi^{-1}(]-\infty, r\right]\right)} \Psi(u)}{r} \leq \frac{\int_{0}^{1} \sup _{|t| \leq \theta} F(x, t) d x+\frac{\mu}{\lambda} G^{\theta}}{\frac{(1-L)}{2} \theta^{2}},
$$

and

$$
\frac{\Psi(w)}{\Phi(w)} \geq \frac{\int_{\frac{3}{4}}^{1} F(x, \delta) d x+\frac{\mu}{\lambda} G(\delta)}{4(1+L) \pi^{4} \delta^{2}\left(\frac{2}{3}\right)^{3}} .
$$

Now if $G(\delta) \geq 0$ then from (11), since $\mu<\eta_{\lambda, g}$ and $\lambda \in \Lambda$ one has

$$
\begin{gathered}
\frac{\int_{0}^{1} \sup _{|t| \leq \theta} F(x, t) d x+\frac{\mu}{\lambda} G^{\theta}}{\frac{(1-L)}{2} \theta^{2}}<\frac{\int_{0}^{1} \sup _{|t| \leq \theta} F(x, t) d x+\frac{\eta_{\lambda, g}}{\lambda} G^{\theta}}{\frac{(1-L)}{2} \theta^{2}}=\frac{1}{\lambda}< \\
\frac{\int_{\frac{3}{4}}^{1} F(x, \delta) d x}{4(1+L) \pi^{4} \delta^{2}\left(\frac{2}{3}\right)^{3}} \leq \frac{\int_{\frac{3}{4}}^{1} F(x, \delta) d x+\frac{\mu}{\lambda} G(\delta)}{4(1+L) \pi^{4} \delta^{2}\left(\frac{2}{3}\right)^{3}} .
\end{gathered}
$$

In the other hand if $G(\delta)<0$ and $G^{\theta}>0$ then

$$
\frac{\int_{0}^{1} \sup _{|t| \leq \theta} F(x, t) d x+\frac{\mu}{\lambda} G^{\theta}}{\frac{(1-L)}{2} \theta^{2}}<\frac{\int_{0}^{1} \sup _{|t| \leq \theta} F(x, t) d x+\frac{\eta_{\lambda, g}}{\lambda} G^{\theta}}{\frac{(1-L)}{2} \theta^{2}} \leq \frac{1}{\lambda}
$$

and also if $G(\delta)<0$ and $G^{\theta}=0$ then we have,

$$
\frac{\int_{0}^{1} \sup _{|t| \leq \theta} F(x, t) d x+\frac{\mu}{\lambda} G^{\theta}}{\frac{(1-L)}{2} \theta^{2}}<\frac{\int_{0}^{1} \sup _{|t| \leq \theta} F(x, t) d x+\frac{\eta_{\lambda, g}}{\lambda} G^{\theta}}{\frac{(1-L)}{2} \theta^{2}}<\frac{1}{\lambda} .
$$

However, the following inequality can be used again.

$$
\frac{1}{\lambda}<\frac{\int_{\frac{3}{4}}^{1} F(x, \delta) d x}{4(1+L) \pi^{4} \delta^{2}\left(\frac{2}{3}\right)^{3}} \leq \frac{\int_{\frac{3}{4}}^{1} F(x, \delta) d x+\frac{\mu}{\lambda} G(\delta)}{4(1+L) \pi^{4} \delta^{2}\left(\frac{2}{3}\right)^{3}} .
$$


So we have shown that the following inequality is established.

$$
\frac{\int_{0}^{1} \sup _{|t| \leq \theta} F(x, t) d x+\frac{\mu}{\lambda} G^{\theta}}{\frac{(1-L)}{2} \theta^{2}}<\frac{1}{\lambda}<\frac{\int_{\frac{3}{4}}^{1} F(x, \delta) d x+\frac{\mu}{\lambda} G(\delta)}{4(1+L) \pi^{4} \delta^{2}\left(\frac{2}{3}\right)^{3}} .
$$

Hence from (11),(12) and (13) we observe that

$$
\frac{\sup _{\Phi(u) \leq r} \Psi(u)}{r}<\frac{1}{\lambda}<\frac{\Psi(w)}{\Phi(w)} .
$$

Hence the condition $\left(a_{1}\right)$ of Theorem 2.1 is satisfied .

Finally, we will show that for every $\lambda \in \Lambda$, functional $\Phi-\lambda \Psi$, is coercive. For this purpose, fix $0<\epsilon<\frac{1-L}{2 \lambda}$. From $\left(A_{3}\right)$ there is a function $K_{\epsilon} \in L^{1}([0,1])$ such that

$$
F(x, t) \leq \epsilon t^{2}+K_{\epsilon}(x),
$$

for every $x \in[0,1]$ and $t \in \mathbb{R}$. Now, for each $u \in X$, we have

$$
\begin{gathered}
\Phi(u)-\lambda \Psi(u) \geq \frac{1}{2}(1-L)\|u\|^{2}-\lambda \int_{0}^{1} F(x, u(x)) d x-\mu G(u(1)) \geq \\
\left(\frac{1-L}{2}-\lambda \epsilon\right)\|u\|^{2}-\lambda \int_{0}^{1} K_{\epsilon}(x) d x-\mu \int_{0}^{u(1)} g(\xi) d \xi .
\end{gathered}
$$

Now if we suppose that $M:=\max _{\xi \in[0,|u(1)|]}|g(\xi)|$, then by using (2) we have ,

$$
\Phi(u)-\lambda \Psi(u) \geq\left(\frac{1-L}{2}-\lambda \epsilon\right)\|u\|^{2}-\lambda\left\|K_{\epsilon}\right\|_{L^{1}[0,1]}-\mu M\|u\|
$$

and thus

$$
\lim _{\|u\| \rightarrow+\infty}(\Phi(u)-\lambda \Psi(u))=+\infty
$$

which means the functional $\Phi-\lambda \Psi$ is coercive, and the condition $\left(a_{2}\right)$ of Theorem 2.1 is verified. Finally, since the weak solutions of the problem (1) are exactly the solutions of the equation $\Phi^{\prime}(u)-\lambda \Psi^{\prime}(u)=0$, Theorem 2.1 ensures the conclusion.

Remark 3.1. In Theorem 3.1 we read $\frac{(1-L) \theta^{2}-2 \lambda \int_{0}^{1} \sup _{|t| \leq \theta} F(x, t) d x}{2 G^{\theta}}=+\infty$ when $G^{\theta}=0$.

In the following, we will express another form of Theorem 3.1, which will be achieved by reversing the role of $\lambda$ and $\mu$.

Theorem 3.2. Assume that there exist two positive constants $\theta, \delta$ with $\theta<\frac{8 \pi^{2}}{3 \sqrt{3}} \delta$, such that $\left(B_{1}\right) 8 \pi^{4}(1+L)\left(\frac{2}{3}\right)^{3} \delta^{2} G^{\theta}<(1-L) \theta^{2} G(\delta) ;$ 
Then, for each

$$
\left.\mu \in \Lambda^{\prime}:=\right] \frac{4(1+L)\left(\frac{2}{3}\right)^{3} \pi^{4} \delta^{2}}{G(\delta)}, \frac{(1-L) \theta^{2}}{2 G^{\theta}}[
$$

and for every $L^{1}$-Carathéodory function $f:[0,1] \times \mathbb{R} \rightarrow \mathbb{R}$ satisfied in conditions of $(A 1)$ and $(A 3)$ of Theorem 3.1 there exists $\eta_{\lambda, g}^{\prime}>0$ where

$$
\eta_{\lambda, g}^{\prime}=\frac{(1-L) \theta^{2}-2 \mu G^{\theta}}{2 \int_{0}^{1} \sup _{|t| \leq \theta} F(x, t) d x}
$$

such that, for each $\lambda \in] 0, \eta_{\lambda, g}^{\prime}[$, the problem (1) admits at least three weak solutions in $X$.

Proof. Fix $\mu \in \Lambda^{\prime}$ and $\left.\lambda \in\right] 0, \eta_{\lambda, g}^{\prime}[$. Let $\hat{\Psi}: X \rightarrow \mathbb{R}$ be defined by

$$
\hat{\Psi}(u)=\frac{\lambda}{\mu} \int_{0}^{1} F(x, u(x)) d x+G(u(1))
$$

for every $u \in X$. It is clearly that

$$
\Phi(u)-\lambda \Psi(u)=\Phi(u)-\mu \hat{\Psi}(u)
$$

for every $u \in X$. Choose $r$ and $w$ as given in (8) and (9).Now, as proof of the Theorem 3.1 , we have

$$
\frac{\hat{\Psi}(w)}{\Phi(w)} \geq \frac{\frac{\lambda}{\mu} \int_{\frac{3}{4}}^{1} F(x, \delta) d x+G(\delta)}{4(1+L) \pi^{4} \delta^{2}\left(\frac{2}{3}\right)^{3}} \geq \frac{G(\delta)}{4(1+L) \pi^{4} \delta^{2}\left(\frac{2}{3}\right)^{3}}>\frac{1}{\mu}
$$

and

$$
\begin{aligned}
& \frac{\sup _{\left.\left.u \in \Phi^{-1}(]-\infty, r\right]\right)} \hat{\Psi}(u)}{r} \leq \frac{\frac{\lambda}{\mu} \int_{0}^{1} \sup _{|t| \leq \theta} F(x, t) d x+G^{\theta}}{\frac{(1-L)}{2} \theta^{2}} \leq \\
& \frac{\frac{\eta^{\prime}{ }_{\lambda, g}}{\mu} \int_{0}^{1} \sup _{|t| \leq \theta} F(x, t) d x+G^{\theta}}{\frac{(1-L)}{2} \theta^{2}}<\frac{1}{\mu} .
\end{aligned}
$$

Therefore from $(16),(17)$ we observe that

$$
\frac{\sup _{\left.\left.u \in \Phi^{-1}(]-\infty, r\right]\right)} \hat{\Psi}(u)}{r}<\frac{1}{\mu}<\frac{\hat{\Psi}(w)}{\Phi(w)} .
$$

On the other hand as proof of the Theorem 3.1 it is easy to show that for every $\mu \in \Lambda^{\prime}$, functional $\Phi-\mu \hat{\Psi}$, is coercive. Hence, since for each $\mu \in \Lambda^{\prime} \subseteq$ ]$\frac{\Phi(w)}{\hat{\Psi}(w)}, \frac{r}{\sup _{\left.\left.u \in \Phi^{-1}(]-\infty, r\right]\right)} \hat{\Psi}(u)}[$ the assumptions of Theorem 2.1 are fulfilled, the conclusion follows from Theorem 2.1 . 
Remark 3.2. In Theorem 3.1 and Theorem 3.2 we can replace the condition $\left(A_{3}\right)$ with the following subquadratic condition:

There exists $\alpha \in(0,2)$ and $M>0$ such that $0<t f(x, t) \leq \alpha F(x, t)$ for all $|t| \geq M$ and $x \in[0,1]$ which is stronger than $\left(A_{3}\right)$ and known as Ambrosetti-Rabinowitz condition. Under this condition by standard computions there is a positive constant $C$ such that $F(x, t) \leq C|t|^{\alpha}$ for all $|t| \geq M$ and $x \in[0,1]$.

Now, we point out the following consequence of Theorem 3.1.

Corollary 3.3. Let $f: \mathbb{R} \rightarrow \mathbb{R}$ be a continuous function. Put $F(t):=\int_{0}^{t} f(\xi) d \xi$ for each $t \in \mathbb{R}$. Fix $\delta>0$ and assume that $F(\delta)>0$ and $F(\xi) \geq 0$ for each $\xi \in[0, \delta]$ and also suppose that

$$
\liminf _{\xi \rightarrow 0} \frac{F(\xi)}{\xi^{2}}=\limsup _{\xi \rightarrow+\infty} \frac{F(\xi)}{\xi^{2}}=0 .
$$

Then, there is $\lambda^{*}>0$ such that for each $\lambda>\lambda^{*}$ and for every nonnegative continuous function $g: \mathbb{R} \rightarrow \mathbb{R}$ there exists $\delta_{\lambda, g}^{\prime}>0$ such that, for each $\mu \in\left[0, \delta_{\lambda, g}^{\prime}[\right.$, the problem

$$
\left\{\begin{array}{l}
u^{(i v)}(x)=\lambda f(u(x))+h(u(x)), \quad x \in[0,1] \\
u(0)=u^{\prime}(0)=0 \\
u^{\prime \prime}(1)=0, \quad u^{\prime \prime \prime}(1)=\mu g(u(1))
\end{array}\right.
$$

admits at least three weak solutions.

Proof. Fix $\lambda>\lambda^{*}:=\frac{16(1+L)\left(\frac{2}{3}\right)^{3} \pi^{4} \delta^{2}}{F(\delta)}$. From the condition

$$
\liminf _{\xi \rightarrow 0} \frac{F(\xi)}{\xi^{2}}=0
$$

there is a sequence $\left.\left\{\theta_{n}\right\} \subset\right] 0,+\infty\left[\right.$ such that $\lim _{n \rightarrow \infty} \theta_{n}=0$ and

$$
\lim _{n \rightarrow \infty} \frac{\sup _{|\xi| \leq \theta_{n}} F(\xi)}{\theta_{n}^{2}}=0 .
$$

Therefore, there exists $\bar{\theta}>0$ such that

$$
\frac{\sup _{|\xi| \leq \bar{\theta}} F(\xi)}{\bar{\theta}^{2}}<\min \left\{\frac{F(\delta)(1-L)}{32(1+L)\left(\frac{2}{3}\right)^{3} \pi^{4} \delta^{2}}, \frac{1-L}{2 \lambda}\right\}
$$

and $\bar{\theta}<\frac{8 \pi^{2}}{3 \sqrt{3}} \delta$. Since $G(\delta)$ is positive hence according to the Theorem 3.1 for each

$$
\mu \in] 0, \delta_{\lambda, g}^{\prime}=\frac{(1-L) \bar{\theta}^{2}-2 \lambda \sup _{|t| \leq \bar{\theta}} F(t)}{2 G^{\bar{\theta}}}[,
$$

problem (18) admits at least three weak solutions. 
Remark 3.3. In the Corollary 3.3, in calculating the upper bound for the $\mu$, if $\lambda$ value increases, then according to (19), the value of $\sup F(t)$, will be reduced. In $|t| \leq \bar{\theta}$

fact, the choice of $\bar{\theta}$, is dependent on the value of $\lambda$. Actually for each fix $\lambda>\lambda^{*}$ we can consider $\bar{\theta}$ such that $\bar{\theta}<\frac{8 \pi^{2}}{3 \sqrt{3}} \delta$ and $(1-L) \bar{\theta}^{2}-2 \lambda \sup _{|t| \leq \bar{\theta}} F(t)>0$.

Now, we present the following example to illustrate corollary 3.3 .

Example 3.4. Consider the problem (1) where

$$
f(t)= \begin{cases}0 & \text { if } t \leq 0 \\ e^{-t^{2}}\left(10 t^{9}-2 t^{11}\right) & \text { if } t>0\end{cases}
$$

and $g(t)=e^{t}$ and $h(t)=0.001$ tanht for all $t \in \mathbb{R}$. We can consider $L=0.001$. Let $\delta=\sqrt{3}$. According to definition of $f$ and $g$ we have,

$$
F(t)= \begin{cases}0 & \text { if } t \leq 0 \\ t^{10} e^{-t^{2}} & \text { if } t>0\end{cases}
$$

and $G(t)=e^{t}-1$.It is clear that

$$
\liminf _{\xi \rightarrow 0} \frac{F(\xi)}{\xi^{2}}=\limsup _{\xi \rightarrow+\infty} \frac{F(\xi)}{\xi^{2}}=0 .
$$

So by applying Corollary 3.3, for every fix

$$
\lambda>\lambda^{*}=\frac{128.128 \pi^{4}}{2187 e^{-3}} \simeq 114.6247
$$

according to $\bar{\theta}<\frac{8 \pi^{2}}{3 \sqrt{3}} \delta$ and $(1-L) \bar{\theta}^{2}-2 \lambda \sup _{|t| \leq \bar{\theta}} F(t)>0$ we can consider $\bar{\theta}$ such that $\bar{\theta}<\frac{8 \pi^{2}}{3 \sqrt{3}} \delta \simeq 26.32$ and $0.999(\bar{\theta})^{2}-2 \lambda \sup _{|t| \leq \bar{\theta}}\left(t^{10} e^{-t^{2}}\right)>0$ and hence for every

$$
\mu \in] 0, \frac{0.999(\bar{\theta})^{2}-2 \lambda \sup _{1}\left(t^{10} e^{-t^{2}}\right)}{2\left(e^{\bar{\theta}}-1\right)}[\text {, }
$$

the problem (1) has at least three weak solutions.

\section{REFERENCES}

[1] Amster, P., and Mariani, M.C., A fixed point operator for a nonlinear boundary value problem, J. Math. Anal. Appl., 266(2001), 160-168.

[2] Bonanno, G., and A. Chinnì, A., Existence of three solutions for a perturbed two-point boundary value problem, Appl. Math. Lett., 23 (2010), 807-811.

[3] Bonanno, G., and D'Aguì, G., Multiplicity results for a perturbed elliptic Neumann problem, Abstract and Applied Analysis 2010 (2010), doi:10.1155/2010/564363, 10 pages. 
[4] Bonanno, G., Chinnì, A., and Tersian, S., Existence results for a two point boundary value problem involving a fourth-order equation, Electronic Journal of Qualitative Theory of Differential Equations, No. 33(2015), 1-9.

[5] Bonanno, G., and Marano, S.A., On the structure of the critical set of non-differentiable functions with a weak compactness condition, Appl. Anal., 89 (2010), 1-10.

[6] Cabada, A., and Tersian, S., Multiplicity of solutions of a two point boundary value problem for a fourth-order equation, Appl. Math. Comput., 24(2011), 1599-1603.

[7] D'Agui, G., Heidarkhani, S., and Molica Bisci, G., Multiple solutions for a perturbed mixed boundary value problem involving the one-dimensional p-Laplacian, Electron. J. Qual. Theory Diff. Eqns., No. 24(2013), 1-14.

[8] Grossinho, M.R., and Ma, T.F., Symmetric equilibria for a beam with a nonlinear elastic foundation, Portugal. Math., 51(1994), 375-393.

[9] Grossinho, M.R., and Tersian, St.A., The dual variational principle and equilibria for a beam resting on a discontinuous nonlinear elastic foundation, Nonlinear Anal., 41(2000), 417-431.

[10] Heidarkhani, S., Khademloo, S., and Solimaninia, A., Multiple solutions for a perturbed fourth-order Kirchhoff type elliptic problem, Portugal. Math. (N.S.), Vol. 71, Fasc. 1 (2014), $39-61$.

[11] Ma, T.F., Positive solutions for a beam equation on a nonlinear elastic foundation, Math. Comput. Modelling, 39(2004), 1195-1201.

[12] Ma, T.F., and da Silva, J., Iterative solutions for a beam equation with nonlinear boundary conditions of third order, Appl. Math. Comput., 159 (2004), 11-18.

[13] Yang, L., Chen, H., and Yang, X., The multiplicity of solutions for fourth-order equations generated from a boundary condition, Appl. Math. Lett., 24(2011), 1599-1603.

[14] Zeidler, E., Nonlinear functional analysis and its applications, Springer, Vol. II. BerlinHeidelberg-New York, 1985. 